\title{
Electromyogram pattern recognition for control of powered upper-limb prostheses: State of the art and challenges for clinical use
}

\author{
Erik Scheme, MSc, PEng; Kevin Englehart, PhD, PEng* \\ Institute of Biomedical Engineering, University of New Brunswick, Fredericton, Canada
}

\begin{abstract}
Using electromyogram (EMG) signals to control upper-limb prostheses is an important clinical option, offering a person with amputation autonomy of control by contracting residual muscles. The dexterity with which one may control a prosthesis has progressed very little, especially when controlling multiple degrees of freedom. Using pattern recognition to discriminate multiple degrees of freedom has shown great promise in the research literature, but it has yet to transition to a clinically viable op tion. This article describes the pertinent issues and best practices in EMG pattern recognition, identifies the major challenges in deploying robust control, and advocates research directions that may have an effect in the near future.
\end{abstract}

Key words: amputee, electromyogram, EMG, linear discriminant analysis, myo electric control, patt ern recognition, prosthetics, rehabilitation, signal processing, upper limb.

\section{INTRODUCTION}

The use of the ele ctromyogram (EMG) as a control source for powered upper-limb prostheses has received considerable attention, because the idea of restoring function by bridging natural neural pathways is a compelling pursuit. The most straight forward and widely used approach to estimating mot or intent is by estimating the intensity of the EMG from electrodes placed on the skin surface, usually placed directly above the remaining muscles that provide the strongest and most stable signal [1]. While basic function can be established in this manner, the corresponding cont rol is seldom intuitive and does not permit effective control of multiple joints in a pros thetic limb. Although myoelectric prostheses have found an important place a s a clinical option in upper -limb prosthetics, the limited dexterity of control is often cited as the primary reason for rather low acceptance of these devices [2].

Conventional myoelectric control schemes use an amplitude measure at each electrode site ( $\mathrm{such}$ as the root-mean-square or mean absolute value of the EMG) to quantify the intensity of contraction in the underlying muscles. Control is achieved by mapping this activity to the required prosthetic function; therefore, it is desirable that these muscles be functionally related to the functions that are to be resto red. If ph ysiologically appropriate muscles are available to res tore lost function, the EMG can be used intuitively, such as when a person with transhumeral amputation controls a prosthetic elbow by using the residual biceps and triceps. In the absence of physiologically appropriate musculature, substitutions must be used, such as using the wrist flexors/extensors to control a hand. If $m$ ore than one device is to be used, mode

\footnotetext{
Abbreviations: $\mathrm{ACE}=$ Acquisition and Control Environment, $\mathrm{AER}=$ active error rate, $\mathrm{AR}=$ autoregressive, $\mathrm{EMG}=$ electromyogram, IMES = Implantable MyoElectric Sensor, LDA = linear discriminant analysis, $\mathrm{TD}=$ time-domain, $\mathrm{TDAR}=$ timedomain/autoregressive, $\mathrm{TER}=$ total error rate, $\mathrm{TMR}=$ targeted muscle reinnervation.

*Address all correspondence to Kevin Englehart, PhD, PEng; Institute of Biomedical Engineering, University of New Brunswick, 25 Dineen Drive, Fredericton, New Brunswick E3B5A3, Canada; 506-458-7020; fax: 506-453-4827. Email: kengleha@unb.ca

DOI:10.1682/JRRD.2010.09.0177
} 
switching is often the only strategy (using a hardware switch or co-contraction) to divert control to an el bow, wrist, or hand. This method of control is, however, slow and counterintuitive.

Many factors contribute to the difficulty of extracting sufficient information from the EMG for dexterous, multifunction control. The most obvious and important is the lack of physiologically a ppropriate musculature from which to estimate the intended motion. This is especially problematic in indivi duals with high-level limb defi ciency, because little or no muscle remains that would produce force in the absent joints.

Another fundamental challenge is a result of the region of muscle activity that is recorded by EMG. The measurable activity is nece ssarily from muscle near the surface of the skin and may involve the contribution of more than one muscle, because of EMG cross talk. When multiple EMG sites are used, muscle co-activation (which is present in most upper-limb articulations) adds another layer of complexity. EMG cross talk, muscle coactivation, and limited sampling depth compromise the ability to estimate dexterous motor intent. Cross talk mixes otherwise independent information sources. Coactivation complicates the task of resolving the intended force about a joint. Limited muscle sampling depth constrains the estimation of inte nt to the activit y of only those muscles near the surface.

The simple one-mus cle one-function approach to conventional control is naïve to the complexities of EMG cross talk, muscle co-activation, and the contribution of deep muscle. This has motivated the use of a patternrecognition approach to my oelectric control. By us ing multiple EMG sites, ef fective feature extract ion, and multidimensional classifiers, one can achieve cont rol of many more classes of motions. While cross talk severely compromises conventional control (the intensity measure is assumed to arise from a single muscle), it is a predictable by-product of the sp atial arrangement in the multi electrode pattern-recognition approach. It may even add information if the "interfering" EMG is not measured by another electrode. The existence of muscle co-activation provides spatial information to a pattern classifier, char-

\footnotetext{
*This impediment is not critical in persons with transradial amputation because, depending on the nature of the deficiency, many of the muscles that naturally control the wrist and hand often remain (with the exception of the intrinsic muscles of the hand).
}

acterizing the natural syner gistic behavior of muscles during a giv en contraction. The contribution of deep muscles can be at least partially utilized by using features that capture low-intensity, low-frequency components of the composite EMG signal.

The use of EMG pattern recognition has shown $g$ reat promise for improv ed dexterity of control in upper-limb prostheses. The concept is by no means new; indeed, the first pattern-recognition-based control schemes were developed as early as the late 1960 s and early 1970 s [3-5]. Improvements in signal processing, multiple-channel instrumentation, and microprocessor technology have facilitated implementation in embedded control systems. Through the efforts of many academic and commercial initiatives, pat tern-recognition-based control appears to be nearing clinical viability. This article describes the current state-of-theart approach to EMG pattern-recognition-based control, categorizes the major challenges in deploying robust con trol, and identifies promising research that may have considerable effect on improving myoelectric control.

\section{BACKGROUND—STATE OF THE ART}

The task of discriminating among a set of muscular contractions for myoelectric control is influenced by the EMG interface and the pattern-recognition methods.

\section{EMG Interface}

The use of EMG as an estimator of motor intent is greatly affected by electrode site selection. ${ }^{\dagger}$ This involves the selection of the $n$ umber and the placement of electrodes. If the availability of useful muscle sites is limited, targeted muscle reinnervation is an effective means of accessing neural information otherwise lost to a person with a high-level amputation, as described below.

\section{Targeted Muscle Reinnervation}

The success of any myoelec tric control scheme is largely determined by the availability of suitable muscu lature from which to extract control information. For the purpose of establishing an intuitive interface, acquiring the control information from physiologically appropriate

\footnotetext{
† Other factors pertaining to the EMG interface may be of minor influence, such as the type of electrode and instrumentation, but these will not be discussed here.
} 
muscles (those that woul $\mathrm{d}$ normally contribute to the function to be restored by the prosthesis) is desirable. Although many physiologically appropriate muscles may be available in the case of persons with transradial amputation [6-7], this becomes a significant problem as the level of amputation becomes more proximal. With higher levels of limb deficiency, restoration of more functionality (devices to control) is required from fewer (or no) physiologically appropriate sites. Even the most sophisticated pattern-recognition approach cannot provide intuitive control without access to the appropriate neural information.

A major advance in the field of upper-limb prosthetics has been the selective transfer of brachial nerves to new muscle sites. This surgical procedure, termed "targeted muscle reinnervation" (TMR), was co nceived by Dr. Todd Kuiken at the Re habilitation Institute of Chicago [8-9]. The most dramatic example of the benefit of TMR in restoring neural pathways is in the case of a person with shoulder disa rticulation amputation. The four major brachial nerves (median, radial, musculocutaneous, ulnar) can be transferred to the pectoral muscles (which are of little functional use to a person with shoulder disarticulation). After the nerves are anastomosed to distinct regions of pectoral tissue, conventional surfa ce electrodes can monitor these restored sources of neural information that no $\mathrm{w}$ correspond to musculature that would have been distal to the amputation (elbow, wrist, and hand), as illustrated in Figure 1.

This procedure has also bee $\mathrm{n}$ effectively applied to persons with transhumeral amputation; the neural infor mation pertaining to hand and wrist function can be restored by transferring the median ne rve to the medial biceps and the dis tal radial nerve to the brachialis or lat eral triceps [10]. In persons with transradial amputation, remnant muscles have been shown to provide good ${ }^{*}$ control of wrist and basic hand function but multiple hand grasps may require TMR to restore the neural intent directed to absent intrinsic muscles of the hand [6].

TMR has been shown to provide dramatic restoration of prosthetic function using conventional control [11] by providing physiologically appropriate control sites. The fact that the remnant nerves are usually truncated at a proximal location along their course (prior to branching)

\footnotetext{
"“Good" in the sense that classification accuracies generally exceed 90 percent.
}

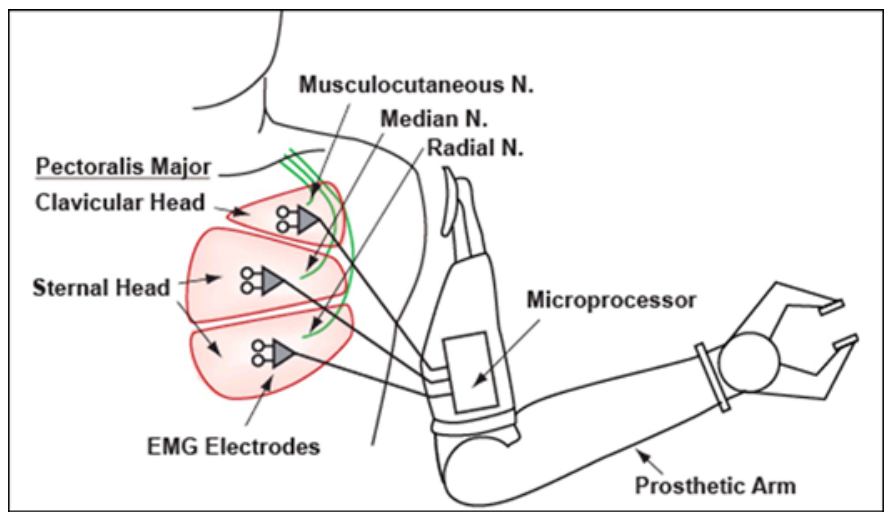

Figure 1.

Targeted muscle reinnervation in person with shoulder disarticulation. $\mathrm{EMG}=$ electromyogram, N. = nerve. Source: Reproduced with permission of the Rehabilitation Institute of Chicago.

results in a mixture of motor functi ons within the fasci cles. The consequence is that the new EMG sites, in general, contain a mixture of functions corresponding to the various branches of each nerve. Through pattern recognition using the TMR sites, these mixtures can be used to discriminate the various functions quite effectively. This was shown by Zhou et al. [10] when a shoulder disarticulation patient was able to discriminate 27 classes of upper-limb function with greater than 97 percent ac curacy. This approach has been shown to perform very well in real-time [12] with both shoulder disarticulation and transhumeral amputees, and clinical experience with pattern recognition and TMR shows great promise.

\section{EMG Site Selection}

Many studies have demon strated that increasing th e number of channels will improve EMG pattern-recognition performance [10,12-15]. For transradial amputees, with electrodes placed above the remnant muscles of the forearm, dramatic improvement in accuracy results when the number of channels is increased up to four or five; beyond this, there are diminishing benefits. Th is effect has been shown in some detail by Hargrove et al. [16]. Six individuals with intact upper limbs performed 10 types of motion (forearm pronation, forearm supination, wrist flexion, wrist extension, radial deviation, ulnar deviation, key grip, chuck grip, hand open, and a rest state). A 16-channel array of equally spaced electrodes was placed around the circumference of the forearm. The effect of selective electrode placement was investigated by comparing naïve site selection (a 
simple symmetric subset of the 16-channel array for 2, 4, 8 , and 16 channels) to "optimal" site selection (the $\mathrm{N}$ sites that yield the highest accuracy for each $\mathrm{N}$-channel subset).

Figure 2 shows that optimum channe 1 selection results in 99 percent accuracy with only four channels. Using the naïve site selec tion scheme, performance climbs quickly to 95 percent with four channels and then gradually increases to 97 pe rcent when using all chan nels. This indicates that, alth ough benefit can be gained by optimizing site selection, it is not a critical factor, provided that a suf ficient number of channe ls is available. The requisite number is not large (nominally four chan nels) in the forearm as the spatial coverage of the EMG appears to be sufficient to charac terize the ava ilable information from the underlying musculature. This was confirmed in this study by comparing the classification accuracy using the EMG from six carefully placed intramuscular sites. No significant difference in accuracy was observed, suggesting that no further discriminant information was available from the EMG.

Although the effect of the numbe $r$ of channels depends on other factors (th e number of classes, th e physiology and geometry of the underlying musculature), these results appe ar to gene ralize well to most studies

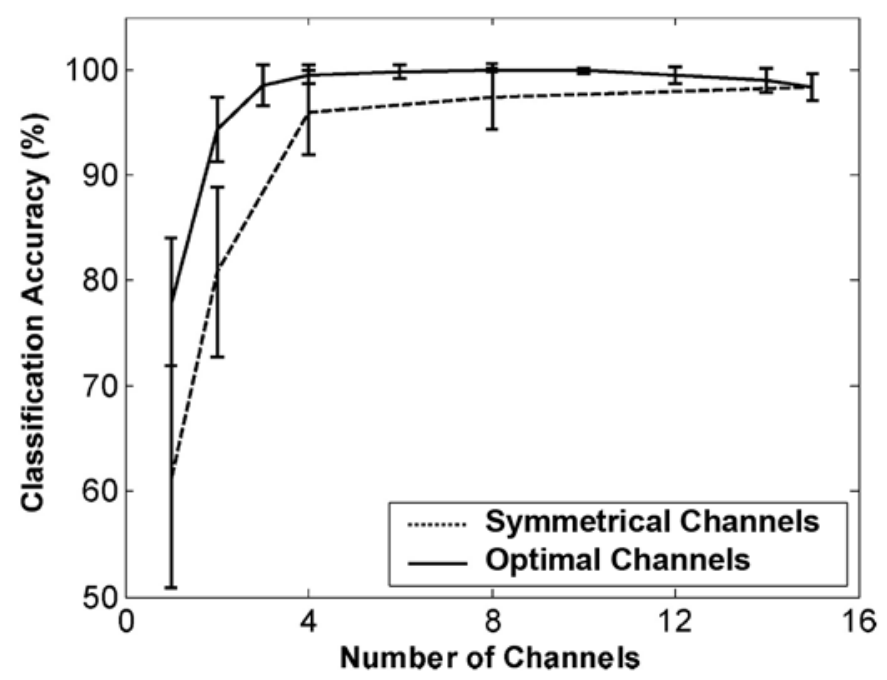

Figure 2.

Increase in classification accuracy as channels are added for both optimal and symmetrical channel subsets. Source: Reprinted with permission of IEEE from Hargrove LJ, Englehart K, Hudgins B. A comparison of surface and intramuscular myoelectric signal classification. IEEE Trans Biomed Eng. 2007;54(5):847-53. [PMID: 17518281] DOI:10.1109/TBME.2006.889192 involving EMG sites on the forearm, as is the case for persons with transradial am putation. For persons with high-level amputations, the required number of channels is not a s straightforward beca use site selection strongly depends on the anatomy of the residual limb or shoulder complex [17]. The required number of channels is very different for TMR us ers. Because of the rich and some what complex information av ailable from nerve transfer sites, as many as 10 ele ctrodes may be required on the pectoralis region and possibly a few other sites for per sons with shoulder disarticulation [10].

\section{Pattern-Recognition Methods}

\section{Best Practices in EMG Pattern Recognition}

All approaches to EMG pattern reco gnition have the fundamental processing stages shown in Figure 3. The EMG signals may be subject to preprocessing to remove unwanted interference; the mos t common sources are power line harmonic s and mo tion artifact due to elec trode movement.

A feature-extraction stage is used to increase the information density of the EMG signals. Ideally, contraction discrimination information should be retained while other irrelevant information is discarded. Many disparate methods of EMG feature extraction have been investigated, including time-domain (TD) [18-21], autoregressive (AR), and cepstral features [13,22 -24]. Joint time-frequency methods have been shown to effectively represent transient EMG patterns resulting from dynamic contractions [2526]. A comparison of feature sets has shown that for slowly varying EMG patterns, a concatenated TD/AR (TDAR) feature set outperform s all others $[16,24]$ but the slight improvement in performance over simple TD fe atures incurs considerable processing overhead.

If the resultant feature set occupies a high-dimensional space (as is $t$ he case wi th most ti me-frequency transforms), it may be beneficial to reduce the dimensionality of the feature space by selecti on of a subset of features $[10,27]$ or a strategic combin ation of features, such as averaging [26], principal components analysis [25,28], independent components analysis [29 ], or nonlinear pro jection [30].

Just as researchers have pursued an extensive range of feature sets, the same can be said of cla ssifiers. Possibly every form of statistical and learning classifier has been investigated and compared for use in myoelectric 


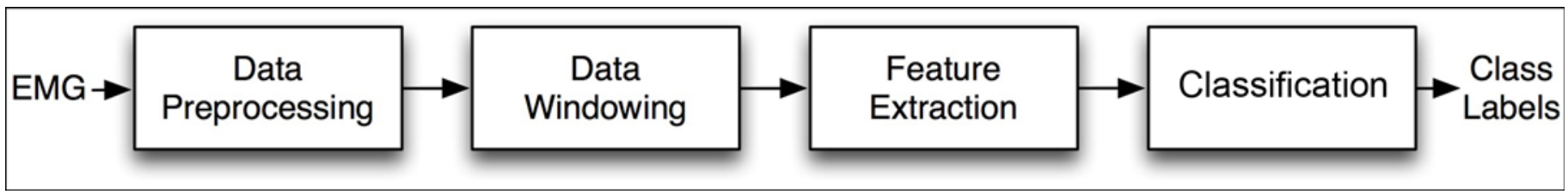

Figure 3.

Stages of signal processing for electromyogram (EMG) pattern recognition.

control. An extensive examination of classifiers is given in Scheme et al. [31] and Zecca et al. [32].

A common observation is that, if a $\mathrm{n}$ effective representation of the EMG signals is in place (an appropriate feature set and a suf ficient number of channels), most modern classifiers will have similar performance. This observation was effectively illustrated by Hargrove et al. [16] in their examination of the relative ef fects of features and classifiers on a co mmon data set (previously described in "EMG Site Selection" section). The effect of the feature set is shown in Figure 4(a), which shows the mean and standard deviation of the c lassification accuracy over the six subjects. The influence of the classifier is shown in Figure 4(b). One can see that the feature representation has greater influence than the complexity of the classifier. The implication is that, with an appropriate feature representation, the classification task is esse ntially a linear problem.

The most popular choices of cla ssifiers in rec ent work are based on marginal advantages in classification performance; these include linear discriminant analysis (LDA) [33], support vector machines [34-37], and hidden Markov models [38-39]. The main advantage of LDA is its simplicity of implementation (especially in an embedded processor) and ease of training.

Before feature extraction, the EMG signals must be windowed; from each window a classification decision will be made. The fundamental trade-off in selecting the window length is that longer windows will improve the stability of the features (reducing the variance and increasing classification performance) but will incur a longer delay in the classification decision.

\footnotetext{
*The delay is almost entirely due to waiting for the data to be acquired. With most feature sets and classifiers, the processing delay is usually inconsequential because modern microprocessors can perform feature extraction and classification in milliseconds.
}

For conventional proportional control, it has been previously accepted that an actuation delay greater than $300 \mathrm{~ms}$ will be perceived by the user. Farrell and Weir. investigated window length for direct control of a hand open/closing task and found $150 \mathrm{~ms}$ to be optimal [40]. The optimum window 1 ength is more difficult to ascertain when pattern recognition is being used, because the relationship among controller delay, classification error, and real-time controllability is complex [41]. Although the classification error tends to de crease with increasing window length [33], increas ed delay deteriorates performance in manipulation. A real-time control test was used to study the interaction between these effects, and the results suggest that the optimum window length for pattern-recognition control is between 150 and $250 \mathrm{~ms}$, depending on the skill of the subject [41].

\section{Classifier Performance Metrics}

Ultimately, the most meanin gful assessment of pros thetic control is an evaluation of the function that a user derives from the device. Several tests of prosthetic function have been developed and are widely used for conventional prosthetics [42-47]. These test s are largely qualitative and are intended to measure skill and efficacy during functional tasks. Given the commercially available prosthetic options, most have ev olved to $b$ e primarily intended for hand manipulation, not multiarticulated tasks involving positioning and orienting a hand using powered wrist, elbow, and shoulder devices. Moreover, they require that a user be fitted with a prosthesis, so conduc ting these tests on subjects before fitting is not possible. The qualitative nature of the tests presents a challenge when one is trying to develop quantitative metrics for control performance. These tests are affected as much by the capabilities of the prosthetic devices; the fit of the socket; and the opinion, skill, and experience of the admitting clinician as they are by the performance of the $\mathrm{c}$ ontrol system. For these reasons, EMG pattern recognition has been measured instead by offline 


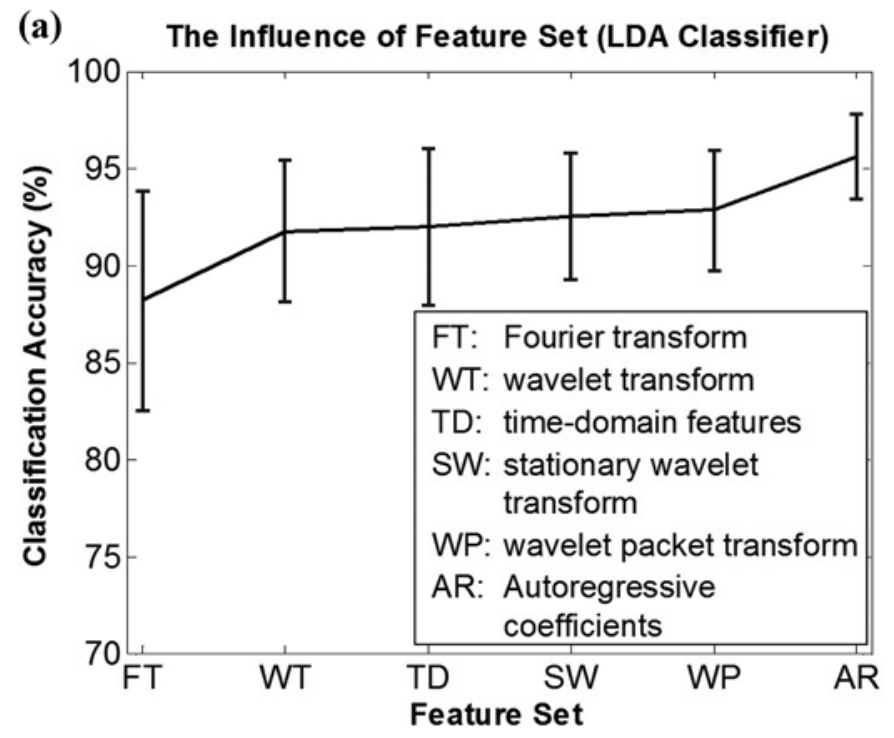

(b) The Influence of the Classifier (AR Features)

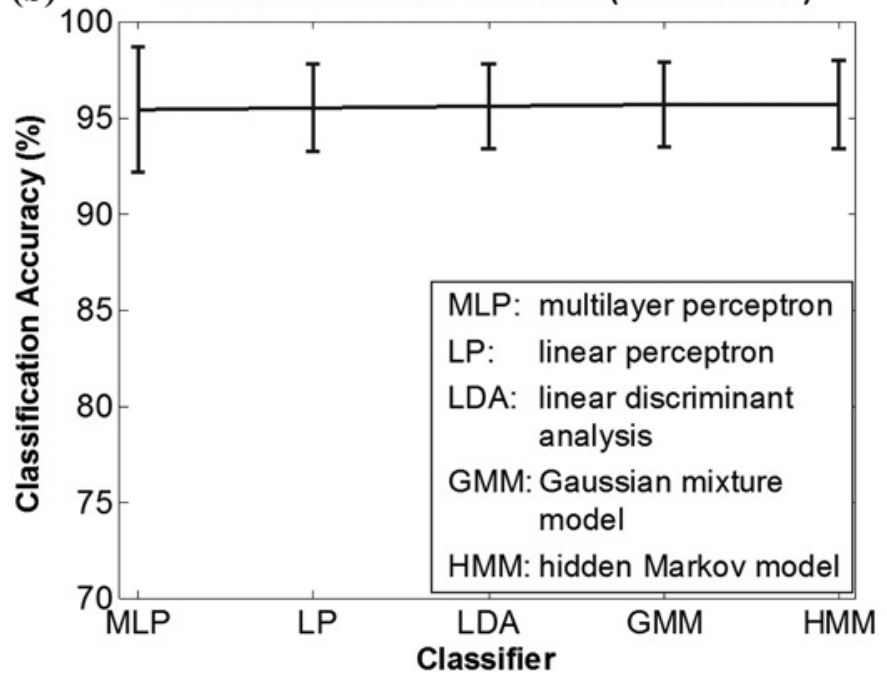

Figure 4.

Comparison of (a) six feature sets using linear discriminant analysis (LDA) classifier and (b) five classifiers using autoregressive (AR) feature set. Source: Reprinted with permission of IEEE from Hargrove LJ, Englehart K, Hudgins B. A comparison of surface and intramuscular myoelectric signal classification. IEEE Trans Biomed Eng. 2007;54(5):847-53. [PMID: 17518281]

DOI:10.1109/TBME.2006.889192.

metrics (largely classification accuracy) and, only more recently, by real-time tests.

Offline Metrics. A recent emphasis on the clinical viability of pattern-recognition-based control has high - lighted a dis parity between classification accuracy and usability (measured using virtual environments and clinical testing). Lock et al. found only a weak correlation between classification accuracy and usability [48]. $\mathrm{H}$ argrove et al. found that they could improve the results of a virtual clothespin placement task while decreasing classification accuracy by including transient contractions in the training data [49]. One possible explanat ion for this paradox is the simplistic manner in which offline classification metrics are defined.

In their expe riments, Hargrove et al. suggeste $d$ that errors causing inadvertent ac tivations of the limb were more "costly" than those th at cause a pause in motion [49]. This may be attributed to the fact that extraneous motions require that the users perform additional corrective motions, increasing frustration and diverging from their originally planned task trajectory. Therefore, defining a metric that directly reflects this contribution to the total classification error is informative. The error rate for purely active decisions, or the active error rate (AER), can be defined as

$$
\mathrm{AER}=100 \% \times \frac{\# \text { Incorrect Active Decisions }}{\text { Total \# Active Decisions }},
$$

where an active decision is a single output from the control system resulting in limb motion. Using this metric in combination with the total error rate (TER), which can be defined as

$$
\mathrm{TER}=100 \% \times \frac{\# \text { Incorrect Decisions }}{\text { Total } \# \text { Decisions }},
$$

may provide a more representative picture of the usability of a clas sifier. Note that deceptively forcing AER to 0 percent is possible by setting all output decisions to no motion; therefore, we recommend always including TER as well (which would tend toward $100 \%$ in this case) when reporting AER.

Real-Time Tests. The desire to provide more mean ingful metrics associated $\mathrm{w}$ ith prosthetic function has motivated the development of real-time tests to assess dynamic performance when pattern recognition is being used. Kuiken et al. introduce d a real-time test involving moving a virtual prosthesis through the range of a selected motion [50]. The efficacy of control is measured by the time required to selec $t$ the desired motion and to complete the desire d motion and the percentage of successfully completed motions. Recently, this paradigm has been extended to multiple degrees of freedom (the Target 
Achievement Control test [51]) to more closely resemble functional assessment.

\section{Persons with Amputation Versus Nondisabled Individuals}

Most investigations of EMG pattern recognition have used individuals with int act limbs instead of those with limb deficiencies. This is often due to the challenges of accessing an appropriate amputee population for research purposes. It is also true that many studies using individuals seek to explore the pattern-recognition performance in an "ideal" context before dealing with the confounding factors introduced by limb deficiency, such as sc ar tissue, variation in muscle geometry, and possible changes in cortical representation and motor pathways [52]. A recent study investigated the performance of variou s classifiers with 10 nondisabled subjects and 5 transradial-amputation subjects [31]. As shown in Figure 5, relatively little difference (within the nondisabled and amputee groups) is seen in the $\mathrm{c}$ lassification error between the better-performing classifiers. What is more interesting, however, is that although the absolute average performance differs between nondisabled and amputee subjects, ${ }^{*}$ the relative performance of the classifiers is fairly consistent between the two groups. That is, the ranking of the classifiers' performance

\footnotetext{
* Obviously, certain factors pertain to persons with amputation that are unique and will influence the absolute results, such as the availability and condition of remnant muscle, scar tissue, and the fit of the socket. Proprioceptive feedback is partially or co mpletely absent when persons with amputation perfo rm contractions related to the missing limb, which can affect the articulation of these contractions.
}
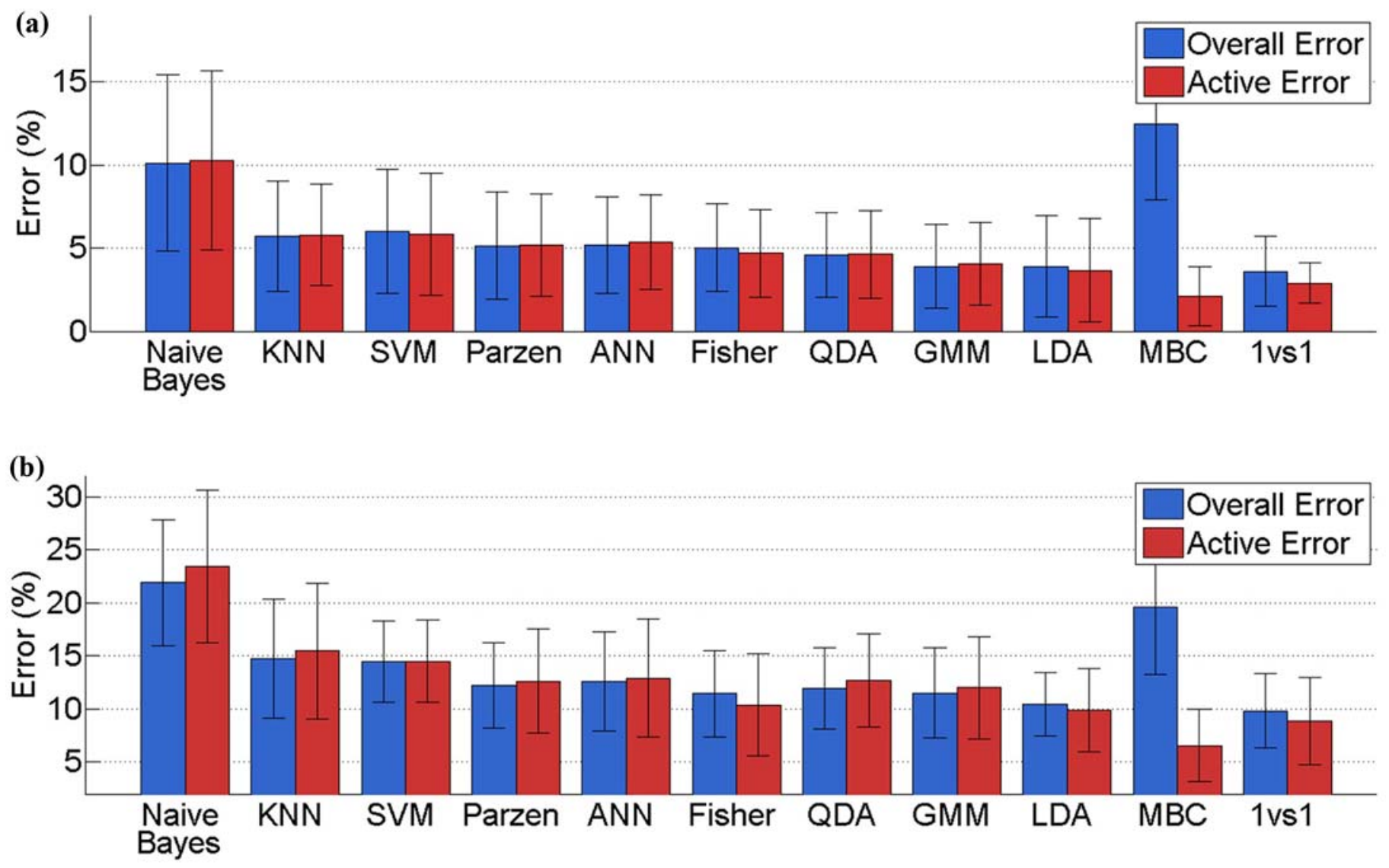

Figure 5.

Comparison of several commonly used classification techniques using conventional classification accuracy. Results represent (a) average over 11 classes of motion collected from 10 nondisabled subjects and (b) average over 7 classes of motion collected from 5 subjects with transradial amputation. ANN = artificial neural network, GMM = Gaussian mixture model, KNN = K nearest neighbor, LDA $=$ linear discriminant analysis, $\mathrm{MBC}=$ multiple binary classifier, $\mathrm{QDA}=$ quadratic discriminant analysis, $\mathrm{SVM}=$ support vector machine. 
is consistent whether from an amputee or a nondisabled population. This result is important because it implies that results from nondisabled individuals can be generalized to persons with amputation.

The same obse rvation can be made when different feature sets are compared, as shown in Figure 6; the rela-
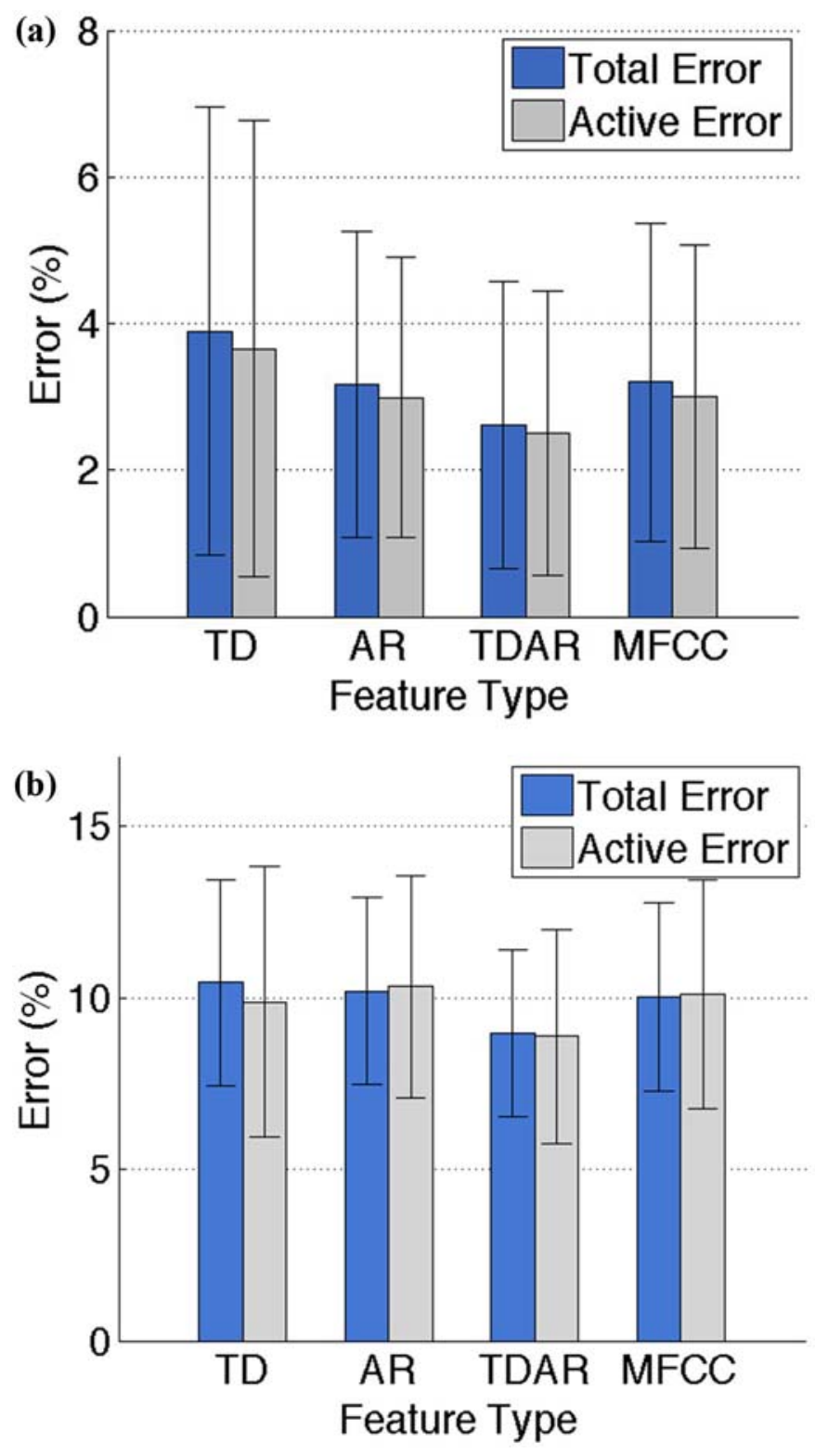

Figure 6.

Comparison of commonly used feature sets. Results represent (a) average over 11 classes of motion collected from 10 nondisabled subjects and (b) average over 7 classes of motion collected from 5 subjects with transradial amputation. $\mathrm{AR}=$ autoregressive, $\mathrm{MFCC}=$ me 1 scale frequency cepstral coefficients, $\mathrm{TD}=$ time-domain, $\mathrm{TDAR}=$ timedomain/autoregressive. tive performance of the feature sets is consistent between nondisabled individuals and persons with amp utation. Again, one can see tha $t$ the TDAR feature set outper forms the others.

\section{Training for Pattern Recognition}

Pattern-recognition-based myoelectric control will very likely s ee clinical implem entation in the near future and will become increasingly stable as the challenges mentioned in the previous section are met. In the near term, it is essential that appropriate tr aining tools and protocols be developed to enable clinical professionals to effectively configure, train, and main tain pattern-recognition-based systems for their clients. Some valuable insight pertaining to the clinical aspects of training users with pattern recognition can be found in Simon et al. [51], Lock et al. [53], Hargrove et al. [54], Stubblefield et al. [55], Nielsen et al. [56], Armiger and Vogelstein [57], Momen et al. [58], and Burger et al. [59].

Software environments for EMG pattern-recognition training are beginning to have an effect on the research community, and the goal is to retool these to be powerful clinical tools for prosthesis configuration, user training, and assessment. The Acquis ition and Control Environ ment (ACE) software package [60] provides a flexible MATLAB-based environment, allowing real-time data acquisition and visualization, control, and configuration of a prosthesis. Pattern recognition as well as conven tional control techniques may be used to drive a prosthetic limb or a simple virtual $\lim b$. The Revolutionizing Prosthetics 2009 project, sponsored by the Defense Advanced Research Projects Agency, produced a po werful Virtual Integration Environment capable of real-time control and interaction in a $v$ irtual environment [61]. Control elements were derived from ACE (implemented in MATLAB), and the virtual environment realized in MSMS (Musculoskeletal Modeling Software), an open-source software environment [62]. Recently, the Rehabilitation Institute of Chicago develo ped a stand-alone software package called CAPS (Control Algorithms for Prosthetics Systems) [12] with a refined user interface and intuitive control configuration, intended for research and clinical deployment. 


\section{MAJOR CHALLENGES TOWARD CLINICAL ROBUSTNESS}

An important question is, What is the required accuracy in order for pattern-recognition control to be considered reliable by the user? The usability of a prosthesis is influenced by ma ny factors, including the fi $t$ of $t$ he socket, the capabilities of th e devices, and the intuiti veness of the control system. In turn, cla ssification accuracy is only one componen $t$ of the intuitiveness and reliability of the controller since, as a metric, it does not consider the dynamic effects of real-time control.

Experience in establishing the relationship between pattern-recognition accuracy and usability is 1 imited, mainly because the dexterous devices required to exploit multifunction control currently only exist in limited numbers as research prototypes. Experiments have been conducted using virtual environments to avoid the need for devices and to focus on the controller itself [12,48-49]. It seems intuitive that higher accuracy should yield control systems with better usability, but preliminary work sug gests that the relationship between accuracy and usability is not so str aightforward [17,49,53]. What is evident, however, is that, as accuracy falls below roughly 85 percent, a multifunction system can become frustrating to use and usability can deteriorate considerably. Accuracy is determined by the capabilities of the user, but (for a reasonable number of classes) an accuracy of 90 percent is a reasonable expectation for most users under ideal conditions.

The reality is, however, that ideal conditions do not exist in practic al use. The most significant issues to be overcome stem from the act of wearing a prosthesis and using it in a functional manner. These, among others, include electrode shift, variation in force, vari ation in position of the limb, and transient changes in EMG.

\section{Electrode Shift}

Each time a user dons a prosthesis, the electrodes will likely settle in a slightly different position, relative to the underlying musculature. The electrodes may also shift during use because of loading and positioning of the limb. A shift of $1 \mathrm{~cm}$ of four electrodes placed circumferentially about the forearm has been shown to increase classification error in a 10-class ex periment from roughly 5 to 20 percent (if shifted distally) and to 40 percent (if rotated about the forearm) [54]. Also, including shifted versions of the data in the training session has been shown to virtu- ally eliminate degradation due to shift in the test set $[54,63]$. A different approach, investigating the effect of bipolar electrode configurations, showed that larger electrodes and wider electrode separation can improve resilience to shift but not nearly as much as incorporating exemplars of shift into the training regimen [64].

Variability in electrode position from day to day may necessitate a daily training session when the prosthesis is being donned. The data from each donning could be stored and reused to emulate the pooling of data from various shift locations. As data are accumulated, the system may become resilient to typical shifts encountered during donning and in use. A daily calibration is probably warranted to accommodate other influences that may affect the EMG, such as electrode impedance (due to skin dryness, humidity), muscle hyper- or hypotrophy, and learning effects as the person with amputation becomes a more experienced user.

\section{Variation in Force}

Conventional control schemes usually map the intensity of a contraction to velocity or position of a device, thereby intrinsically incorporating a natural variation in contraction strength. Patte rn-recognition control, however, relies on clustering repeatable patterns of EMG activity into discernible classes. Contractions performed at different force levels may be very different from one another and therefore prese nt a challenge to a pattern classifier. To demonstrate the impact of variation in force, we conducted an experiment ${ }^{*}$ in which users performed contractions at 20 to 80 percent of the strongest contraction they felt comfortable producing (this is clearly a subjective rating). Using eight electrodes spaced about the forearm, 11 subjects were asked to produce nine classes of motion (wrist flexion, wrist extens ion, wrist pronation, wrist su pination, hand open, key grip, chuck grip, power grip, and pinch grip). A tenth class of no movement was also included. Each of the nine active classes was performed at each of the prescribed levels of relative effort.

To investigate the ability of a pattern-recognition system to handle variations in force, we traine d a classifier by using data from each force level and tested it at each level. The results of the 10-class experiment using a TD feature set and an LDA classifier are shown in Figure 7.

\footnotetext{
*These are unpublished results acquired for illustration in this review.
} 


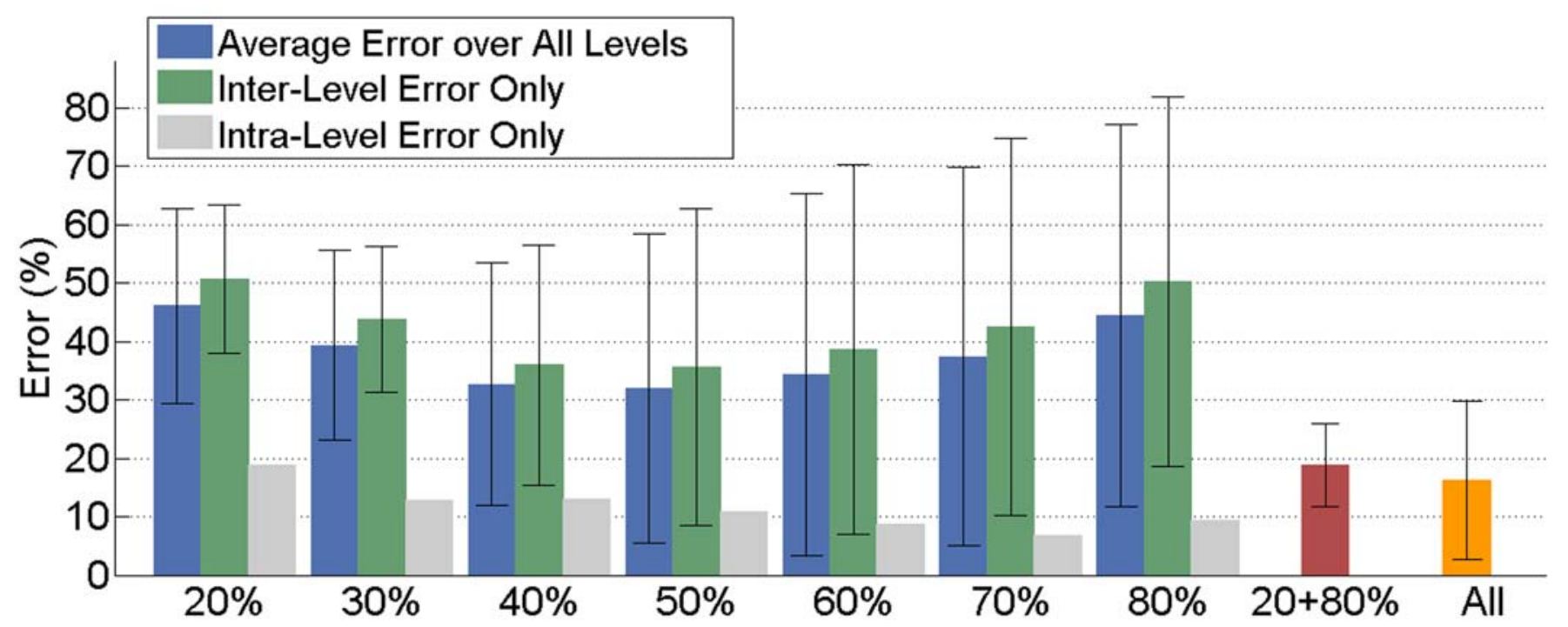

Figure 7.

Effects of training and testing with different force levels. Blue bars represent training at specified force levels (horizontal axis) and testing with all force levels, green bars when testing only at force levels different from training set, and gray bars when testing at same force level as training.

As would be expected, training and testing a classifier at the same force level results in the best ac curacies (gray bars). The best intralevel accuracy is achieved in the 60 to 70 percent effort range, which may be explained by the fact that subjects generally find it di fficult to produce low-level contractions with consistency. High-level contractions sometimes produce tremor, which may degrade recognition.

To determine the ability of a pattern-recognition system to genera lize to new force levels not seen during training, we trained classifiers at each force level and then tested them on all force levels. The blue bars show the error when tested on contractions from all force levels. Clearly, the prese nce of contra ctions from unse en force levels increases the error considerably, to the point where the system would be unusable (gre ater than $32 \%$ error). The green bars show the recognition error on only the unseen force levels (interlevel error); as expected, this performance is worst of all.

In an attempt to counteract this severe degradation in performance, an obvious strategy is to include exemplars from all force levels in the training set. The orange bar labeled "All" indicates the performance when we pooled data from all force levels and tested on all levels. It is encouraging that the error dr ops substantially to 17 per cent, which is not ideal, but approaching a usable system.
It is not desirable, however, to have persons with amputation undergo an extensive training s ession that would require contractions from all levels, especially if they are to do this daily to accomm odate electrode shift while donning the prosthesis. A restricted protocol was therefore investigated: using only the lowest (20\%) and highest $(80 \%)$ force levels. As shown in the figure (indicated by a red ba $r$ ), the error incre ases only mar ginally (to $19 \%$ ) from training with all levels. W ork is ongoing to further reduce the intralevel errors to levels that are comfortably within a range of a usable system $(<10 \%$ error $)$.

\section{Variation in Position of Limb}

The unrestricted functional use of an uppe r-limb prosthesis necessitates using the limb in a variety of positions. For a person with transradial or transhume ral amputation, this will i mpose a loading of the muscles inside of the socket from which EMG is being recorded. This will alter the nature of the EMG due to compression of the muscle and, possibly, elicitation of eccentric con traction or mechanical stimulation of the muscle . As well, various limb positions will impose different gravitational forces, causing displacement of the muscles.

Scheme et al. performed an experiment to characterize the influence of limb position on pattern-recognition accuracy [65]. EMG data corres ponding to eight classes o $f$ 
motion were collected from eight healthy nondisabled subjects (seven male, one female). The subjects were fitted with a cuf $f$ made of thermoformable gel (taken from a $6 \mathrm{~mm}$ Alpha liner by Ohio W illow Wood; Mt. S terling, Ohio) that was embedded with eig ht equally spaced pairs of stainless steel dome electrodes. The cuff was placed around the dominant forearm proximal to the elbow at the position with largest muscle bulk. When a $\mathrm{c}$ uff is us ed, only the effect of muscle displacement due to gravity will influence the EMG; c ompression at the electrode sites only occurs when a socket is used.

Subjects were prompted to produce contractions corresponding to the following eight classes of motion: wrist flexion/extension, wrist pronation/supination, hand open, power grip, pinch grip, and a no motion/rest class. Each repetition was sustained for 3 seconds and a 3 second rest was given between subsequent repe titions. This was repeated twice in each of the limb pos itions shown in Figure 8.

TD features and an LDA class ifier were used to process the data. In a manner similar to the force experiment, the data were trained at each individual position and tested at each individual position. The results are illustrated in Figure 9.

As expected, the intraposition error is low, averaging 6.9 percent over all positions. The worst intraposition error was observed at P7 (h umerus reaching forward, elbow bent at $90^{\circ}$ ); the best is at $\mathrm{P} 3$ (straight arm hanging at side). The average interposition error (35\%) is considerably worse than the average intraposition error. Indeed, almost none of the situations of interposition classifica tion yield clinically ac ceptable performance: the lowest error is 13.6 pe rcent when training with arm straight down and testing when leaning down (these should intuitively be similar); the next lowest is 17.7 percent.

As with force and electrode shift, a strategy to reduce interposition error is to train with exemplars from each position. Figure 10 shows that this is an ef fective approach, yielding an average error of 7.4 percent, which is only slightly higher than the average intraposition error of 6.9 percent.

Using these same data, Scheme et al. have shown that the results can be slightly further improved by instrumenting the humerus and fore arm with accelerometers [65-66]. Whether accelerometers are included or only EMG is used, however, the burden remains on the person with amputation to complete a training se ssion with an exhaustive set that includes all positions.

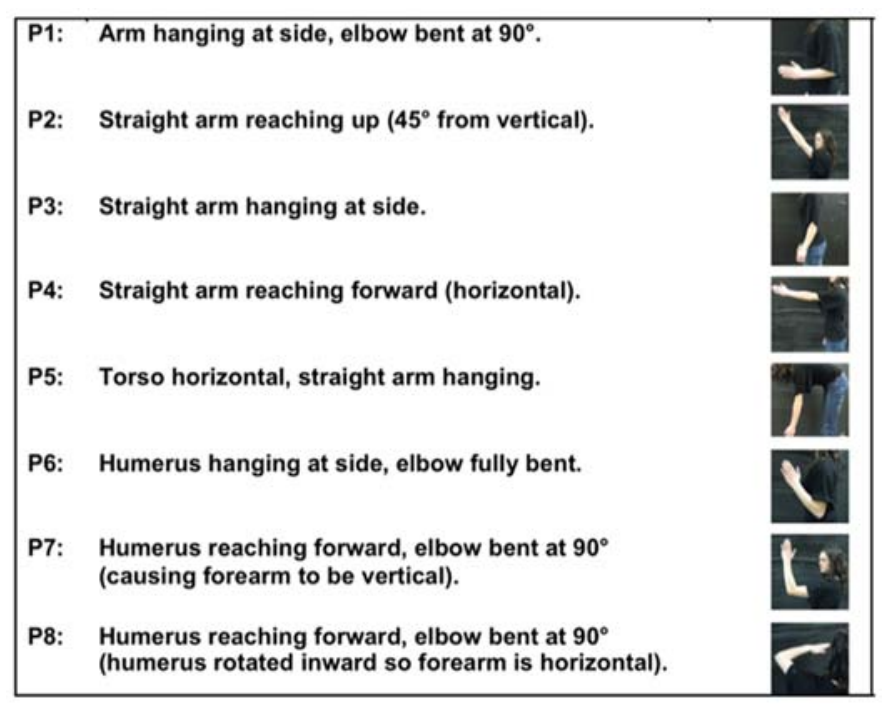

Figure 8.

Limb positions (labeled $\mathrm{P} 1-\mathrm{P} 8$ ) in which subjects re peated contractions corresponding to wrist flexion/extension, wrist pronation/supination, hand open, power grip, pinch grip, and no motion/rest.

\section{Transient Changes in EMG}

Transient changes are additional factors that con found the use of EMG and are a result of short- and longterm variations in the recording environment during use. External interference, electrode impedance changes, electrode shift and lift, and muscle fatigue may alter the EMG and present challenges to clinical robustness.

Strategic filtering and electromagnetic shielding can eliminate most forms of interference. The other sources of variation are intrinsic to the system and cannot be effectively suppressed and are therefore more problem atic. Although a pattern-recognition system may be cali brated upon donning, the nature of th ese effects is unpredictable, and therefore, the system must adapt to the changes in EMG. Adaptive EMG pattern-recognition systems have been investigated by several groups [67-70]. Adaptation of a pa ttern classifier is challenging because the system must know not only how to ad apt but also when to adapt. For a classifier to know how to adapt properly, it must have knowledge (or a reliable es timate) of the intended motion that generates the data to which it must adapt. If the user is directed (supervised) during the data acquisition, the classes will be known and the task is straightforward. The great est benefits to be gained through adaptation, however, are du ring normal, unsupervised use, and therefore, the system must gues $s$ the 


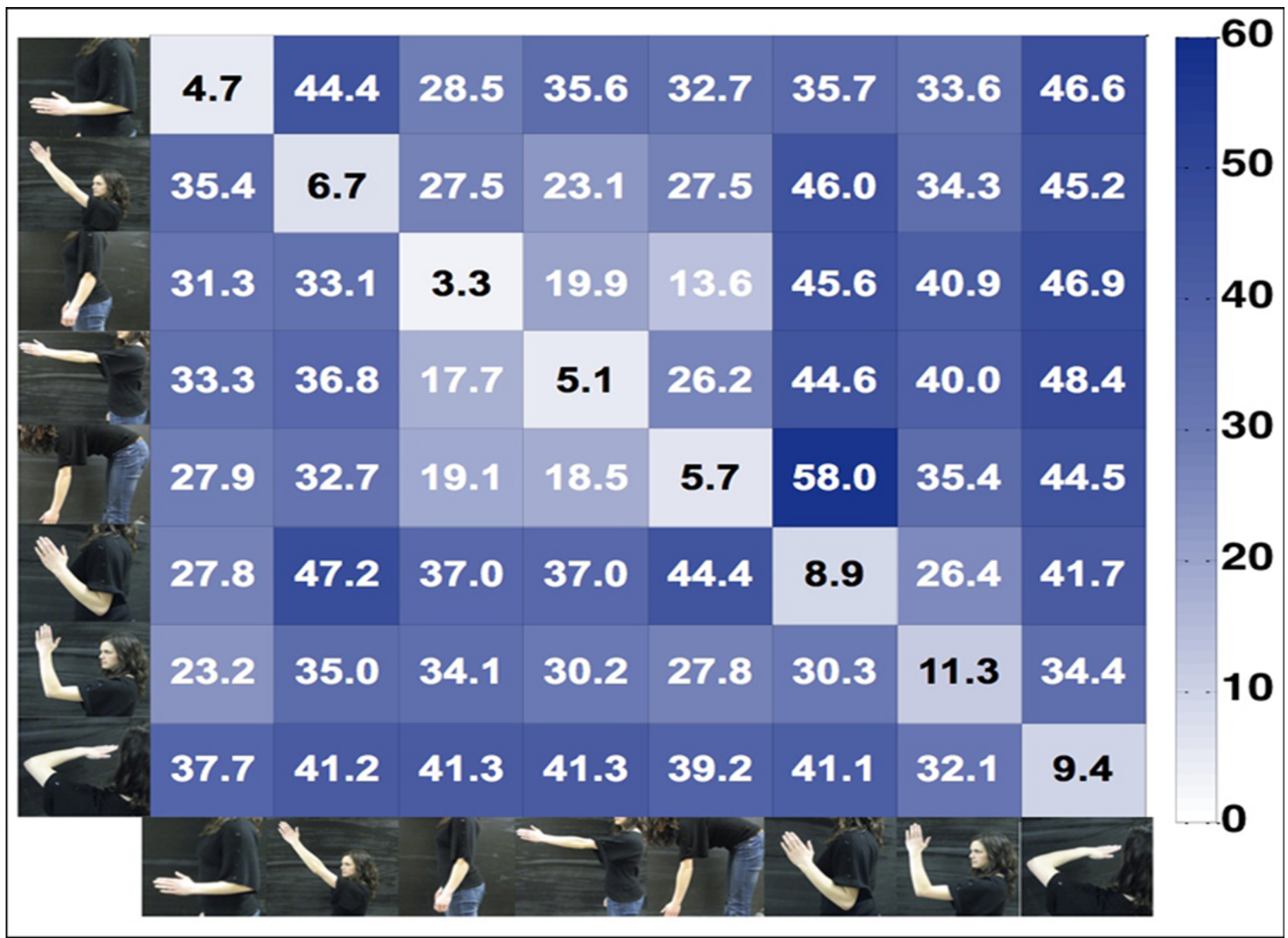

Figure 9.

Intra- and interposition error. Rows indicate position from which training data were acquired. Main diagonal represents intraclass errors.

intended class. Knowing when to adapt is equally critical. When parsing a data stream in real time, the system must be confident that the data are representative of the estimated class. Some success has been reported through the use of entropy measures to characterize the uncertainty in the data and, therefore, attempt to select appropriate data for retraining. Improvement as a result of adaptation has been shown during extended use [67] and for specifically induced effects (electrode shift, fatigue) [70], but the dynamics of adaptation and the interaction with the user are still not well understood. A completely stable, unsupervised solution has yet to be realized but is of great clinical interest.

\section{FUTURE PROSPECTS}

The main clinical advantage of surface EMG is that it is noninvasive, but its robustness is limited by inherent problems of electrode movement and lift, skin impedance changes over the day, and motion artifact. Implanted or intramuscular EMG (us ing wire electrodes) circu mvents these issues and has been shown to produce similar pattern-recognition results as EMG, as long as the intra muscular EMG is impl anted in the appropriat e muscles $[16,71]$. It is neither conveni ent nor practical, though, to use percutaneous wires in chronic use, due to the risk of breakage and infection. 


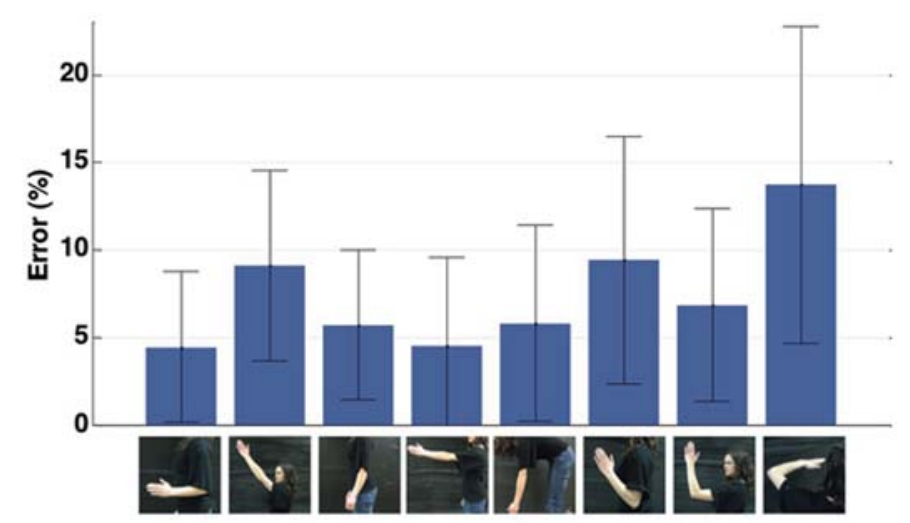

Figure 10.

Error when testing at each position (horizontal axis), and training with data from all positions.

Wireless, implanted EMG sensors have recently become available as resea rch prototypes [72] and may incorporate the functional a dvantages of wire elec trodes with minimal inva siveness. Implantable MyoElectric Sensor (IMES) electrodes [73] are $2 \mathrm{~mm}$ in diameter and $15 \mathrm{~mm}$ long with a recording electrode on both ends. The devices are powered inductively and transmit EMG data using an inductive link between an external coil and a coil within the device. These devices have been successfully used to decode finger motion in a primate by implantation of nine IMESs in the forearm of a macaque monkey [74].

In addition to improved recording robustness, another advantage of embedded EMG sensors is the prospect of providing simultaneous control of multiple degrees of freedom. Although the re have be en attempts to accomplish this with EMG [56,75-77], none of the se approaches have been found to be clinically reliable. It is unlikely that robust, simulta neous, independent control of multiple degrees of free dom is attainable for the per son with transradial ampu tation using surfa ce EMG because of the complex nature of forearm muscle synergies [78-79], the inherent cross talk in the surface signal $[75,80]$, and the displacement in these muscles that occurs during c ontraction. Various approaches have attempted to decompose the multimuscle syner gies into the activity of constituent muscles, but the models are very sensitive to anatomical and electrophysiological factors that may change during use. While more feasible, achieving simultaneous, independent control using intramuscular EMG is still a challenging task, because a sta ble muscle synergy map must be developed for each user.
There is currently intens e international research focused on the recording of motor intent from peripheral nerves and the motor cortex for brain-machine interfaces, functional electrical stimulation, and prosthetic control. Although great advances have been made in these fields, there remain many medic al and technical challenges to overcome before they are rea dy for clinical consideration. An excellent overview of this work is provided in Velliste et al. [81], Micera et al. [82], and Baker [83].

\section{CONCLUSIONS}

The capabilities and limitations of EMG pattern recognition for prost hetic control are wel 1 understood in research settings and are now under scrutiny in the context of clinical applicat ion. This is of great interest in terms of functional benefit to users, especially now that highly dexterous, multifunc tion hands and wr ists are commercially available. This article has described the most important aspects of pattern-recognition-based control, which include the EMG interface, signal processing, and performance evaluation. The major issues that must be accommodated to yield reliable performance in rea 1 use have been identified and illustrated, and methods for mitigating these issues have been described. The near future will be a transitional period for EMG control, as more clinical experience guides best practices for interfacing, training, and signal processing. Recent developments in intramuscular and neural sensors will one day have a dramatic effect on the field, but only when medical and technical challenges are met.

\section{ACKNOWLEDGMENTS}

\section{Author Contributions:}

Study concept and design: E. Scheme, K. Englehart.

Acquisition of data: E. Scheme.

Analysis and interpretation of data: E. Scheme, K. Englehart.

Drafting of manuscript: E. Scheme, K. Englehart.

Obtained funding: K. Englehart.

Financial Disclosures: The authors have declared that no competing interests exist.

Funding/Support: This material was based on work supported by the Natural Sciences and Engineering Research Council of Canada (Discovery Grant 217354-10), the National Institutes of Health (grant 5R01HD058000-03), and the Atlantic Innovation Fund. 


\section{REFERENCES}

1. Parker P, Englehart K, Hudgins B. Myoelectric signal processing for control of $\mathrm{p}$ owered limb prostheses. J Electromyogr Kinesiol. 2006;16(6):541-48. [PMID: 17045489] DOI:10.1016/j.jelekin.2006.08.006

2. Atkins DJ, Heard DCY, Donovan WH. Epidemiologic overview of individuals with upper-limb loss and their reported research priorities. J Prosthet Orthot. 1996;8(1):2-11. DOI:10.1097/00008526-199600810-00003

3. Finley FR, Wirta RW. Myocoder studies of multiple myopotential response. Arch Phys Med Rehabil. 1967;48(11): 598-601. [PMID: 6060789]

4. Lawrence P, Herberts P, Kadefors R. Experiences with a multifunctional hand prosthesis controlled by myoelectric patterns. In: Gav rilovic MM, Wilson $\mathrm{AB}$ Jr, editors. Advances in external control of human extremities. Belgrade (Yugoslavia): Etan; 1973. p. 47-65.

5. Lyman JH, Freedy A, Prior R. Fundamental and applied research related to the design and development of upper-limb externally powered prostheses. Bull Prosthet Res. 1976;13: $184-95$.

6. Li G, Kuiken TA. EMG pattern recognition control of multifunctional prostheses by transradial amputees. Conf Proc IEEE Eng Med Biol Soc. 2009;2009:6914-17. [PMID: 19964455]

7. Li G, Schultz AE, Kuiken TA. Quantifying pattern recognition-based myoelectric control of multifunctional transradial prostheses. IEEE Trans Neural Syst Rehabil Eng. 2010;18(2):185-92. [PMID: 20071269]

8. Hijjawi JB, Kuiken TA, Lipschutz RD, Miller LA, S tubblefield KA, Dumanian GA . Improved myoelectric prosthesis control accomplished using mu ltiple nerve transfers. Plast Reconstr Surg. 2006;118(7):1573-78. [PMID: 17102730] DOI:10.1097/01.prs.0000242487.62487.fb

9. Kuiken TA, Miller LA, Lipschutz RD, Lock BA, Stubblefield K, Marasco PD, Zhou P, Dumanian GA. T argeted reinnervation for enhanced prosthetic arm function in a woman with a proximal amputation: A case study. Lancet. 2007;369(9559):371-80. [PMID: 17276777]

DOI:10.1016/S0140-6736(07)60193-7

10. Zhou P, Lowery MM, Englehart KB, Huang H, Li G, Hargrove L, Dew ald JP, Kuiken TA. Decoding a new neural machine interface for control of artificial limbs. J Neurophysiol. 2007;98(5):2974-82. [PMID: 17728391]

DOI:10.1152/jn.00178.2007

11. Kuiken TA, Dumanian GA, Lipschutz RD, Miller LA, Stubblefield KA. The use of tar geted muscle reinnervation for improved myoelectric prosthesis control in a bilateral shoulder disarticulation amputee. Prosthet Orthot Int. 2004; 28(3):245-53. [PMID: 15658637]
12. Kuiken TA, Li G, Lock BA, Lipschutz RD, Miller LA, Stubblefield KA, Englehart KB. Targeted muscle reinnervation for real-time myoelectric con trol of multifunction artificial arms. JAMA. 2009;301(6):619-28. [PMID: 19211469] DOI:10.1001/jama.2009.116

13. Doerschuk PC, Gustafson DE, Willsky AS. Upper extremity limb function discrimination using EMG signal analysis. IEEE Trans Biomed Eng. 1983;30(1):18-29. [PMID: 6826182] DOI:10.1109/TBME.1983.325162

14. Kuruganti U, Hudgins B, Scott RN. Two-channel enhancement of a multifunction control system. IEEE $\mathrm{T}$ rans Biomed Eng. 1995;42(1):109-11. [PMID: 7851924] DOI:10.1109/10.362912

15. Englehart K, Hudgin B, Parker PA. A wavelet-based continuous classification scheme for multifunction myoelectric control. IEEE Trans Biomed Eng. 2001;48(3):302-11. [PMID: 11327498] DOI:10.1109/10.914793

16. Hargrove LJ, Engleh art K, Hudgins B. A com parison of surface and intramuscular myoelectric signal classification. IEEE Trans Biomed Eng. 2007;54(5):847-53. [PMID: 17518281] DOI:10.1109/TBME.2006.889192

17. Losier Y, Englehart K, Hudgins B. Evaluation of shoulder complex motion-based input strategies for en dpoint prosthetic-limb control using a dual-task paradigm. J Rehabil Res Dev. 2011;48(6):669-78. DOI:10.1682/JRRD.2010.08.0165

18. Lee S, Saridis G. The control of a prosthetic arm by EMG pattern recognition. IEEE Trans Auto Control. 1984;29(4): 290-302.

19. Hudgins B, Parker P, Scott RN. A new strategy for multifunction myoelectric control. IEEE T rans Biomed Eng. 1993; 40(1):82-94. [PMID: 8468080] DOI:10.1109/10.204774

20. Barniv Y, Aguilar M, Hasanbelliu E. Using EMG to anticipate head motion for vi rtual-environment applications. IEEE Trans Biomed Eng. 2005;52(6):1078-93. [PMID: 15977737] DOI:10.1109/TBME.2005.848378

21. Zardoshti-Kermani M, Wheeler BC, Badie K, Hashemi RM. EMG feature evaluation for $m$ ovement control of upper extremity prostheses. IEEE $\mathrm{T}$ rans Rehabilitation Eng. 1995;3(4):324-33. DOI:10.1109/86.481972

22. Graupe D, Salahi J, Kohn KH. Multifunctional prosthesis and orthosis control via microcomputer identification of temporal pattern differences in single-site myoelectric signals. J Biomed Eng. 1982;4(1):17-22. [PMID: 7078136] DOI:10.1016/0141-5425(82)90021-8

23. Kang WJ, Shiu JR, Cheng CK, Lai JS, Tsao HW, Kuo TS. The application of cepstral coefficients and maximum likelihood method in EMG p attern recognition. IEEE T rans 
Biomed Eng. 1995;42(8):777-85. [PMID: 7642191]

DOI:10.1109/10.398638

24. Huang Y, Englehart KB, Hudgins B, Chan AD. A Gaussian mixture model based classification scheme for myoelectric control of powered upper limb prostheses. IEEE Trans Biomed Eng. 2005;52(11):1801-11. [PMID: 16285383] DOI:10.1109/TBME.2005.856295

25. Englehart K, Hudgins B, Parker PA, Stevenson M. Classification of the myoelectric signal using time-frequency based representations. Med Eng Phys. 1999;21(6-7):431-38. [PMID: 10624739] DOI:10.1016/S1350-4533(99)00066-1

26. Lucas MF, Gaufriau A, Pascual S, Doncarli C, Farina D. Multi-channel surface EMG classification u sing support vector machines and signal-based wavelet optimization. Biomed Signal Process Control. 2008;3(2):169-74. DOI:10.1016/j.bspc.2007.09.002

27. Nazarpour K, Sharafat AR, Firoozabadi SMP. Surface EMG signal classification using a selective mix of higher order statistics. Conf Proc IEEE Eng Med Biol Soc. 2005; 4:4208-11. [PMID: 17281162]

28. Hargrove LJ, Li G, Englehart KB, Hudgins BS. Principal components analysis preprocessing for improved classification accuracies in pattern-recognition-based myoelectric control. IEEE Trans Biomed Eng. 2009;56(5):1407-14. [PMID: 19473932] DOI:10.1109/TBME.2008.2008171

29. Naik GR, Kumar DK, Palaniswami M. Surface EMG based hand gesture identification using semi blind ICA: Validation of ICA matrix analysis. Electromyogr Clin Neurophysiol. 2008;48(3-4):169-80. [PMID: 18551837]

30. Gallant PJ, Morin EL, Peppard LE. Feature-based classification of myoelectric signals using artificial neural $n$ etworks. Med Biol Eng Comput. 1998;36(4):485-89.

[PMID: 10198534] DOI:10.1007/BF02523219

31. Scheme E, Englehart K, Hudgins B. A one-versus-one classifier for improved robustness of myoelectric control. The 18th Congress of the International Society of Electrophysiology and Kinesiology; 2010 Jun 16-19; Aalborg, Denmark.

32. Zecca M, Micera S, Carrozza M, Dario P. Control of multifunctional prosthetic hands by processing the electromyo graphic signal. Crit Rev Biomed Eng. 2002;30(4-6):459-85. [PMID: 12739757] DOI:10.1615/CritRevBiomedEng.v30.i456.80

33. Englehart K, Hu dgins B. A robu st, real-time control scheme for multifunction myoelectric control. IEEE Trans Biomed Eng. 2003;50(7):848-54. [PMID: 12848352] DOI:10.1109/TBME.2003.813539

34. Winkler M, Boschmann A, Kaufmann P, Platzner M. Towards multi-movement hand prostheses: combining adaptive classification with high precision socket. Proceed- ings of th e 2nd European Conference on Technically Assisted Rehabilitation; 2009 Jan 30-31; Berlin, Germany.

35. Kaufmann P, Englehart K, Platzner M. Fluct uating EMG signals: Investigating long-term effects of pattern matching algorithms. Conf Proc IEEE Eng Med Bi ol Soc. 20 10; 2010:6357-60. [PMID: 21096692]

36. Oskoei MA, Hu H. Support vector machine-based classification scheme for myoelectric control applied to upper limb. IEEE Trans Biomed Eng. 2008;55(8):1956-65. [PMID: 18632358] DOI:10.1109/TBME.2008.919734

37. Yang D, Zhao J, Gu Y, Jiang L, Liu H. EMG pattern recognition and grasping force estimation: Improvement to the myocontrol of multi-DOF prosthetic hands. Proceedings of the 2009 IEEE/RSJ International Conference on Intelligent Robots and Systems; 2009 Oct 11-15; St. Louis (MO); Piscataway (NJ): IEEE Press; 2009. p. 516-21.

38. Chan AD, Englehart KB. Continuous myoelectric control for powered prostheses using hidden Markov models. IEEE Trans Biomed Eng. 2005;52(1):121-24.

[PMID: 16561571]

DOI:10.1109/TBME.2004.836492

39. Li Y, Chen X, Tian J, Zhang X, Wang K, Yang J. Automatic recognition of sign language subwords based on portable accelerometer and EMG sensors. Proceedings of the International Conference on Multimodal Interfaces and th $e$ Workshop on Machine Learning for Mul timodal Interaction; 2010 Nov 8-12; Beijing, China; New York (NY): ACM; 2009.

40. Farrell TR, Weir RF. The optimal controller delay for myoelectric prostheses. IEEE Trans Neural Syst Rehabil Eng. 2007;15(1):111-18. [PMID: 17436883]

DOI:10.1109/TNSRE.2007.891391

41. Smith LH, Lock BA, Hargrove L. Effects of window length and classification accuracy on the real-time controllability of pattern recognition myoelectric control. Proceedings of the 18th Congress of the International Society for Electrophysiology and Kinesiology; 2010 Jun 16-19; Aalborg, Denmark.

42. Wright FV, Hubbard S, Jutai J, Naumann S. The Prosthetic Upper Extremity Index: Development and reliability testing of a new functional status questionnaire for children who use up per extremity prostheses. J Hand Ther. 2001; 14(2):91-104. [PMID: 11382260]

43. Hermansson LM, Fisher AG, Bernspång $\mathrm{B}$, Eliass on AC. Assessment of capacity for myoelectric control: A new Rasch-built measure of prosthetic hand control. J Rehabil Med. 2005;37(3):166-71. [PMID: 16040474]

44. Sanderson ER, Scott RN. UNB Test of Prosthetic Function: A test for unilateral upper extremity amputees, ages 2-13; Fredericton (Canada): University of New Brunswick; 1985. 
45. Kopp B, Kunkel A, Flor H, Platz T, Rose U, Mauritz KH, Gresser K, McCulloch KL, Taub E. The Arm Motor Ability Test: Reliability, validity, and sensitivity to change of an instrument for assessing disability in activities of daily living. Arch Phys Med Rehabil. 1997;78(6):615-20.

[PMID: 9196469] DOI:10.1016/S0003-9993(97)90427-5

46. Wolf SL, Catlin PA, Ellis M, Archer AL, Morgan B, Piacentino A. Assessing Wolf motor function test as outcome measure for research in patients after stroke. Stroke. 2001; 32(7):1635-39. [PMID: 11441212]

47. Light CM, Chappell PH, Kyberd PJ. Establishing a standardized clinical assessment tool of path ologic and p rosthetic hand function: Normative data, reliability, and validity. Arch Phys Med Rehabil. 2002;83(6):776-83. [PMID: 12048655]

48. Lock BA, Englehart K, Hudgins B. Real-time myoelectric control in a virtual environment to relate usability vs. accuracy. Proceedings of the 2005 MyoElectric Controls/Powered Prosthetics Symposium; 2005 Aug 17-19; Fredericton, Canada. Available from: http://dukespace.lib.duke.edu/ dspace/bitstream/handle/10161/2721/

Lock 01.pdf? sequence $=3$

49. Hargrove L, Losier Y, Lock B, Englehart K, Hudgins B. A real-time pattern recognition based $\mathrm{m}$ yoelectric control usability study implemented in a virtual environment. Conf Proc IEEE Eng Med Biol Soc. 2007;2007:4842-45. [PMID: 18003090]

50. Kuiken TA, Li G, Lock BA, Lipschutz RD, Miller LA, Stubblefield KA, Englehart KB. Targeted muscle reinnervation for real-time myoelectric control of multifunction artificial arms. JAMA. 2009;301(6):619-28.

[PMID: 19211469]

DOI:10.1001/jama.2009.116

51. Simon AM, Hargrove LJ, Lock BA, Kuiken T A. A decision-based velocity ramp for minimizing the effect of misclassifications during real-time pattern recognition control, submitted to IEEE Trans Biomed Eng. Forthcoming 2011.

52. Montoya P, Ritter K, Huse E, Larbig W, Braun C, Töpfner S, Lutzenberger W, Grodd W, Flor H, Birbaumer N. The cortical somatotopic map and phantom phenomena in subjects with congenital limb atrophy and traumatic amputees with phantom limb pain. Eur J Neurosci. 1998;10(3):1095-1102. [PMID: 9753177]

DOI:10.1046/j.1460-9568.1998.00122.x

53. Lock B, Engl ehart K, Hud gins B. VR tools for devel opment and training of advanced prosthetics. Proceedings of the 11th Annual CyberTherapy Conference; 2006 Jun 1215; Ottawa, Canada.

54. Hargrove L, Englehart K, Hudgins B. The effect of electrode displacements on pattern recognition based myoelectric control. Conf Proc IEEE Eng M ed Biol Soc. 2006;
1:2203-6. [PMID: 17946096]

DOI:10.1109/IEMBS.2006.260681

55. Stubblefield KA, Miller LA, Lip schutz RD, Kuiken T A. Occupational therapy protocol for am putees with targeted muscle reinnervation. J Rehabil Res Dev. 2009;46(4):481-88. [PMID: 19882483]

DOI:10.1682/JRRD.2008.10.0138

56. Nielsen JL, Holmgaard S, Jiang N, Englehart K, Farina D, Parker PA. A training strategy for simultaneous and proportional force control of multifunction myoelectric prostheses for unilateral upper-limb amputees. IEEE $\mathrm{T}$ rans Biomed Eng. 2010;99:1-8.

57. Armiger RS, Vogelstein RJ. Air-Guitar Hero: A real-time video game interface for training and evaluation of dexterous upper-extremity neuroprosthetic control algorithms. Proceedings of the IEEE Biomedical Circuits and Systems Conference;2008 Nov 20-24; Baltimore, MD. Los Alamitos (CA): IEEE; 2008. p. 121-24.

58. Momen K, Krishnan S, Chau T. Real-time classification of forearm electromyographic signals corresponding to userselected intentional movements for multifunction prosthesis control. IEEE Trans Neural Syst R ehabil Eng. 2007; 15(4):535-42. [PMID: 18198711]

DOI:10.1109/TNSRE.2007.9

59. Burger H, Brezovar D, Marinek C. Comparison of clinical test and questionnaires for the evaluation of upp er limb prosthetic use in chi ldren. Disabil Rehabil. 2004;26(1415):911-16. [PMID:15497921] DOI:10.1080/09638280410001708931

60. Scheme E, Englehart K. A fl exible user interface for rapid prototyping of advan ced real-time myoelectric control schemes. Proceedings of the MyoElectric Controls/Powered Prosthetics Symposium; Fredericton, New Brunswick, Canada; 2008 Aug 13-15.

61. Bishop W, Armiger R, Burck J, Bridges M, Hauschild M, Englehart K, Scheme E, Vogelstein RJ, Beaty J, Harshbarger S. A real-time virtual integration environment for the design and develo pment of neural prosthetic systems. Conf Proc IEEE Eng Med Biol Soc. 2008;2008:615-19. [PMID: 19162731]

62. Davoodi R, Urata C, Todorov E, Loeb GE. Development of clinician-friendly software for musculoskeletal modeling and control. Conf Proc IEEE Eng Med Biol Soc. 2004;6: 4622-25. [PMID: 17271337]

63. Hargrove L, Englehart K, Hudgins B. A training strategy to reduce classification degradation due to electrode displacements in pat tern recognition based my oelectric control. Biomed Signal Process Control. 2008;3(2):175-80. DOI:10.1016/j.bspc.2007.11.005

64. Young A, Hargrove L. Strategies to reduce myoelectric pattern recognition sensitivity to electrode shift. Proceedings of the 18th Congress of the International Society for Elec- 
trophysiology and Kinesiology; 2010 Jun 16-19. Aalborg, Denmark.

65. Scheme E, Fougner A, Stavdahl Ø, Chan AC, Englehart K. Examining the adverse ef fects of limb position on pattern recognition based myoelectric control. Conf Proc IEEE Eng Med Biol Soc. 2010;2010:6337-40. [PMID: 21097173]

66. Fougner A, Scheme E, Chan AD, Kyberd PJ, Englehart K, Stavdahl Ø. Adverse effects of limb position on myoelectric control of up per-limb prostheses. Proceedings of the 22nd International Conference of the Society for Medical Innovation and T echnology; 2010 Sep 2-4; Trondheim, Norway.

67. Fukuda O, Tsuji T, Kaneko M, Otsuka A. A human-assisting manipulator teleoperated by EMG signals and arm motions. IEEE T rans Robotics Automation. 2003;19(2): 210-22. DOI:10.1109/TRA.2003.808873

68. Sensinger JW, Lock BA Kuiken TA. Adaptive pattern recognition of myoelectric signals: Exploration of conceptual framework and practical algorithms. IEEE Trans Neural Syst Rehabil Eng. 2009;17(3):271-78. [PMID: 19497834]

69. Kato R, Fujita T, Yokoi H, Arai T. Adaptable EMG prosthetic hand using on-line learning method: Investigation of mutual adaptation between human and adaptable machine. The 15th I EEE International Symposium on Robot and Human Interactive Communication, 2006. 2006 Sep 6-8; Los Alamitos (CA): IEEE. p. 599-604.

70. Biron KB, Englehart K. EMG pattern recognition adaptation. Proceedings of the 18th Congress of the International Society of Electroph ysiology and Kinesiology; 2010 Jun 16-19; Aalborg, Denmark.

71. Farrell TR, Weir RF. A comparison of the effects of electrode implantation and targeting on pattern classification accuracy for prosthesis control. IEEE Trans Biomed Eng. 2008;55(9):2198-11. [PMID: 18713689] DOI:10.1109/TBME.2008.923917

72. Weir RF, Troyk P, Demichele G, Kerns D. Technical details of the implantable myoelectric sensor (IMES) system for multifunction prosthesis control. Conf Proc IEEE Eng Med Biol Soc. 2005;7:7377-40. [PMID: 17281975]

73. Weir RF, Troyk PR, Demichele G, Kerns D, Schorsch JF, Maas H. Implantable myoelectric sensors (IMES) for prosthesis control: Development and testing. IEEE Trans Biomed Eng. 2009;56(1):159-71.

DOI:10.1109/TBME.2008.2005942

74. Baker JJ, Scheme E, Englehart K, Hutchinson DT, Greger B. Continuous detection and decoding of dexterous finger flexions with implantable myoelectric sensors. IEEE Trans Neural Syst Rehabil Eng. 2010;18(4):424-32. [PMID: 20378481]

75. Jiang N, Englehart KB, Parker PA. Extracting simultaneous and proportional neural control information for multipleDOF prostheses from the surface electromyographic signal.
IEEE Trans Biomed Eng. 2009;56(4):1070-80.

[PMID: 19272889]

DOI:10.1109/TBME.2008.2007967

76. Smith RJ, Tenore F, Huberdeau D, Etienne-Cummings R, Thakor NV. Continuous decoding of finger position from surface EMG signals for the control of powered prostheses. Conf Proc IEEE Eng Med Biol Soc. 2008;2008:197-200. [PMID: 19162627]

77. Yatsenko D, McDo nnall D, G uillory KS. Si multaneous, proportional, multi-axis prosthesis control using multichannel surface EMG. Conf Proc IEEE Eng Med Biol Soc. 2007;2007:6134-37. [PMID: 18003415]

78. Ajiboye AB, Weir RF. Muscle synergies as a $\mathrm{p}$ redictive framework for the EMG patterns of new hand postures. J Neural Eng. 2009;6(3):036004. [PMID: 19436081] DOI: $10.1088 / 1741-2560 / 6 / 3 / 036004$

79. Overduin SA, d'Avella A, Roh J, Bizzi E. Modulation of muscle synergy recruitment in primate grasping. J Neurosci. 2008;28(4):880-92. [PMID: 18216196]

DOI:10.1523/JNEUROSCI.2869-07.2008

80. Lowery MM, Weir RF, Kuiken TA. Simulation of intramuscular EMG signals detected using implantable myoelectric sensors (IMES). IEEE Trans Biomed Eng. 2006; 53(10):1926-33. [PMID: 17019856]

DOI:10.1109/TBME.2006.881774

81. Velliste M, Perel S, Spalding MC, Whitford AS, Schwartz AB. Cortical control of a prosthetic arm for self-feeding. Nature. 2008;453(7198):1098-1101. [PMID: 18509337] DOI:10.1038/nature06996

82. Micera S, Carpaneto J, Raspopo vic S. Cont rol of hand prostheses using peripheral information. IEEE Rev Biomed Eng. 2010;3:48-68.

83. Baker JJ. D exterous finger movements: Decoding neuroand myoelectric signals and properties of fing er-related neurons in $\mathrm{m}$ otor cortex [dissertation]. [Salt Lake $\mathrm{C}$ ity (UT)]: University of Utah; 2010.

Submitted for publication September 10, 2010. Accepted in revised form February 28, 2011.

This article and any supplementary material should be cited as follows:

Scheme E, Englehart K. Electromyogram pattern recognition for control of powered upper-limb prostheses: State of the art and challenges for clinical use. J Rehabil Res Dev. 2011;48(6):643-60.

DOI:10.1682/JRRD.2010.09.0177

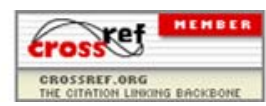


